\title{
UNILATERAL PSOAS MINOR: A CASE REPORT
}

\author{
Krasimir Markov ${ }^{1}$, Mihaela Zaharieva ${ }^{1}$, Pamela Dragieva ${ }^{1}$, Yordan Kozhuharov ${ }^{1}$, \\ Denitsa Nikolova ${ }^{1}$, Radina Deltcheva ${ }^{1}$, George St. Stoyanov ${ }^{2,3}$ \\ ${ }^{1}$ Student, Faculty of Medicine, Medical University of Varna \\ ${ }^{2}$ Department of Anatomy and Cell Biology, Faculty of Medicine, \\ Medical University of Varna \\ ${ }^{3}$ Department of Physiology and Pathophysiology, Faculty of Medicine, \\ Medical University of Varna
}

\begin{abstract}
INTRODUCTION: The posterior abdominal wall is a complex region of human anatomy. It is formed by the lumbar vertebrae, pelvic girdle and the five main posterior abdominal muscles - the iliacus, psoas major, quadratus lumborum, the diaphragm, their associated fascia and the variable psoas minor and tertius muscles. The psoas muscle group is comprised of the long fusiform muscles - major, minor and tertius, with only the psoas major muscle being an obligatory muscle present in all individuals. The psoas muscle group, however, is extremely variable and the muscles have differences not only in their structure and morphology, but also in their innervation. The psoas minor is an extremely variable inconstant muscle, a prime example for a vestigial structure in the human body.

CASE REPORT: During a standard posterior abdominal wall dissection of an adult female cadaver a unilateral right-sided psoas minor muscle was established, with a complete absence of the same muscle on the contralateral left side. The unilateral right-sided psoas minor muscle was located superficially and laterally to the psoas major muscle; it was fusiform in shape and its body measured $96 \mathrm{~mm}$ in length and $16 \mathrm{~mm}$ in circumference.

CONCLUSION: The psoas muscle group is one of the most variable muscle groups in the human body. It could be bilateral - located on both sides of the vertebral column on the anterior surface of psoas major muscle. Our case is representative of one of the not so common variations of the psoas minor muscle with muscle morphology extremely representative of this type of unilateral variation.
\end{abstract}

Keywords: psoas minor, posterior abdominal wall, variations, morphology

Address for correspondence:

George S. Stoyanov

Department of Anatomy and Cell Biology

Medical University of Varna

55 Marin Drinov St

9002 Varna, Bulgaria

e-mail: georgi.geesh@gmail.com

Received: October 21, 2017

Accepted: November 17, 2017

\section{INTRODUCTION}

The posterior abdominal wall is a complex region of anatomy. It is formed by the lumbar vertebrae, pelvic girdle and the posterior abdominal muscles - the iliacus, psoas major, quadratus lumborum, their associated fascia and the variable psoas minor and tertius muscles (1). Topographically a portion of the diaphragm also resides on the posterior abdominal wall, although it does not per se belong to this muscle group. 
The psoas muscle group is comprised of long fusiform muscles - major, minor and tertius, with only the psoas major muscle being an obligatory muscle present in all individuals. The muscles from the psoas muscle group, however, have differences not only in their structure and morphology, but also in their innervation (1).

The psoas major is a constant muscle in the human body. It is divided into a deep and superficial part $(1,2)$. The deep part originates from the transverse processes of the lumbar vertebrae (L) - L1 to L5. The superficial part originates from the lateral surfaces of the last thoracic (Th) and the first four L vertebrae - Th12-L4 and their neighboring intervertebral discs. Innervation of the psoas major is through the anterior rami of L1 to L3 nerves (2). Its function is flexion of the hip and lateral flexion of the vertebral column. In extremely rare instances a small bundle of muscle fibers can arise directly from variable places of the psoas major muscle, forming the psoas accessorius muscle, often confused with the psoas minor muscle, although in some instances the accessorius and minor muscles can fuse their bodies or tendons $(3,4)$.

The psoas minor is an inconstant muscle. It originates as vertical fascicles inserted on the bodies of the Th12 and L1 vertebrae (5). It is innervated from the anterior rami of the $\mathrm{L} 1 \mathrm{nerve}$. This variable muscle is a prime example for a vestigial structure in the human body. Although its actions include bending of the lumber spine and stabilization of the hip joint, these actions are extremely limited and the absence of the muscle does not reflect on them in any manner. In rare instances the muscle can be present only unilaterally.

The psoas tertius is the least studied muscle of the psoas group as its presence is extremely rare in humans (6). The muscle is extremely variable in its places of origin and there have been several reports of extremely variable morphology, some with a clinical manifestation due to the muscle penetrating and splitting the femoral nerve (6).

The psoas muscle group is not per se an individual group of muscle, as it can also be viewed as part of the ileopsoas muscle, together with the iliacus muscle, due to the joining together of their tendons (1).

\section{CASE REPORT}

This study was carried out at the Department of Anatomy and Cell Biology, Medical University - Varna "Prof. Dr. Paraskev Stoyanov", Varna, Bulgaria.

During a standard posterior abdominal wall dissection of an adult female cadaver a unilateral right-sided psoas minor muscle was established, with a complete absence of the same muscle on the contralateral left side (Fig. 1).

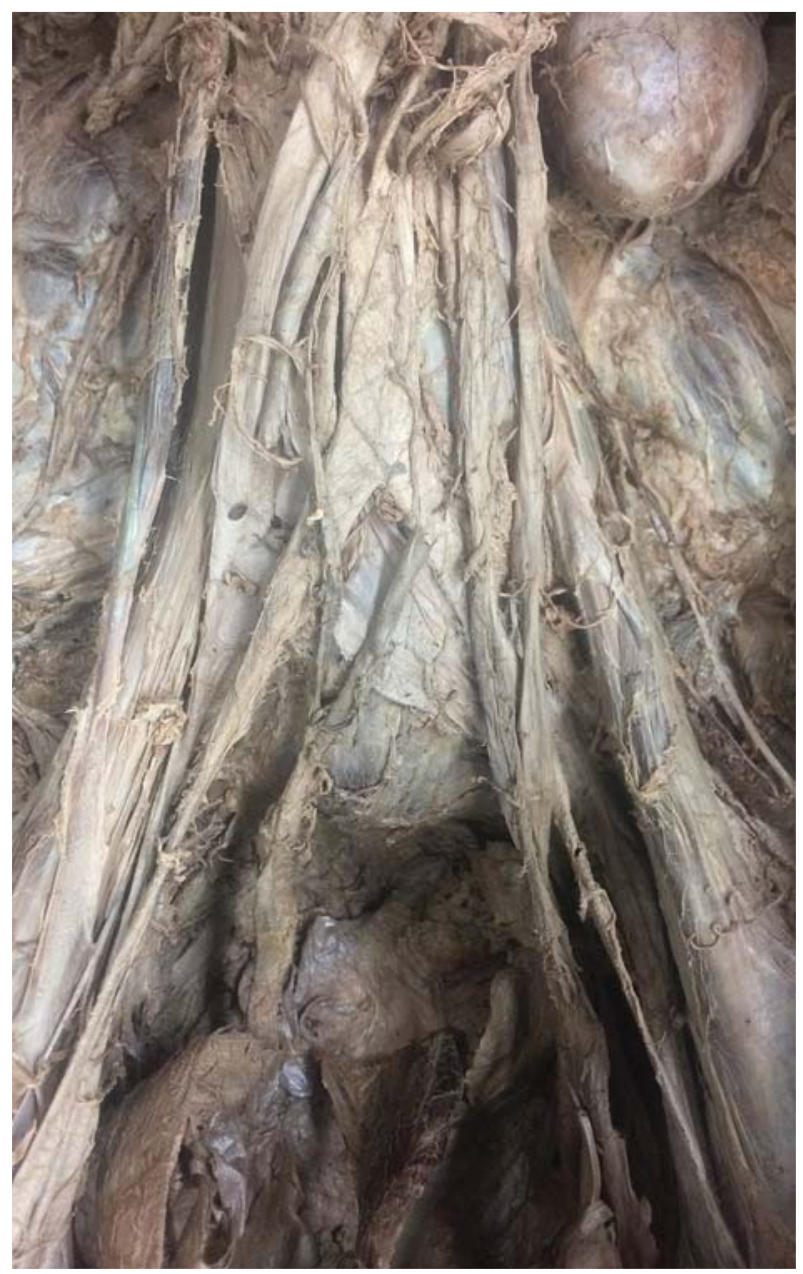

Fig. 1. View of the posterior abdominal wall with a unilateral right-sided psoas minor muscle

The unilateral right-sided psoas minor muscle was located superficially and laterally to the psoas major muscle (Fig. 2). The muscle was fusiform in shape and its body measured $96 \mathrm{~mm}$ in length and 16 $\mathrm{mm}$ in circumference. The muscle tendon was dilated distally and inserted into the ileopectineal arch. 
Krasimir Markov, Mihaela Zaharieva, Pamela Dragieva et al.

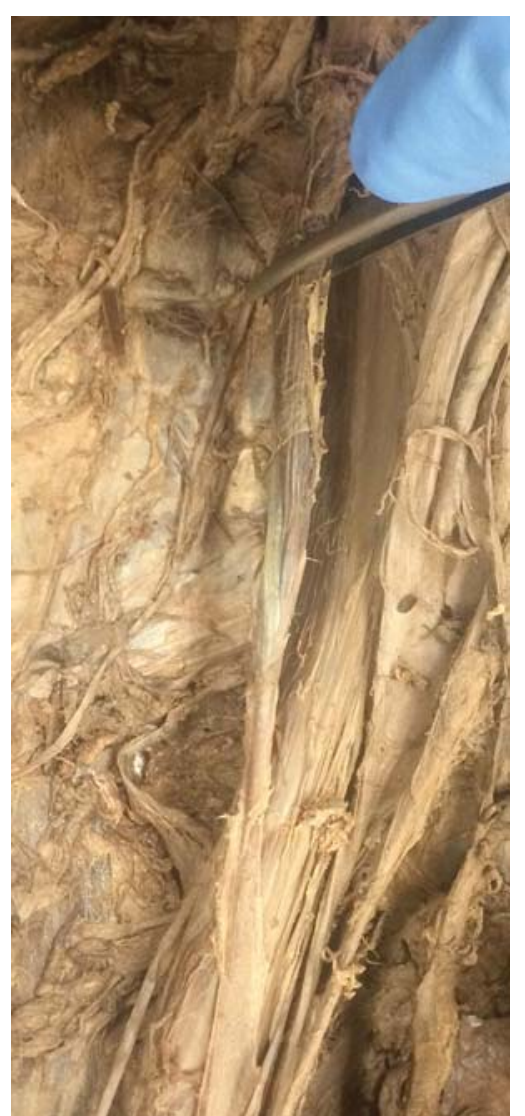

Fig. 2. View of the unilateral right-sided psoas minor muscle. Note the muscle body has been manually retracted

\section{DISCUSSION}

The psoas muscle group is one of the most variable muscle groups in the human body. It could be bilateral - located on both sides of the vertebral column on the anterior surface of psoas major muscle (2). It could be absent on both sides. In rare cases it could be present unilaterally - only on the one side, left or right, of the vertebral column (5).

Another variation of the psoas minor muscle could be due to gender and racial differences. For example, there were many reported cases of prevalence of psoas minor in Japanese population which ranges from $35 \%$ to $55 \%$ (7). However, other research showed that the prevalence of psoas minor between young black and white men and found that psoas minor was absent in $91 \%$ of African-Americans and in only $13 \%$ of white, non-Hispanic Americans $(4,8)$. The psoas minor muscle is also more frequently absent in females, regardless of side or race $(4,8)$.

\section{CONCLUSION}

Our case is representative of one of the not so common variations of the psoas minor muscle with muscle morphology extremely representative of this type of unilateral variation.

\section{Acknowledgements}

The authors would like to thank the reviewers of Scripta Scientifica Vox Studentium for their reviews, recommendations and suggestions, which represent an important contribution to the final from of the text.

\section{Conflict of Interest}

The authors declare no conflict of interest.

\section{REFERENCES}

1. Jelev L, Shivarov V, Surchev L. Bilateral variations of the psoas major and the iliacus muscles and presence of an undescribed variant muscle - accessory iliopsoas muscle. Ann Anat. 2005;187(3):2816. doi: 10.1016/j.anat.2004.10.006

2. Protas M, Voin V, Wang JM, Iwanaga J, Loukas M, Tubbs RS. A Rare Case of Double-Headed Psoas Minor Muscle with Review of its Known Variants. Cureus. 2017; 9(6):e1312. doi: 10.7759/cureus.1312

3. Joshi SD, Joshi SS, Dandekar UK, Daini SR. Morphology of Psoas Minor and Psoas Accessorius. J Anat Soc India. 2010;59(1):31-4. doi: 10.1016/ S0003-2778(10)80008-5

4. Hanson P, Magnusson SP, Sorensen $\mathrm{H}$, simonsen EB. Anatomical differences in the psoas muscles in young black and white men. J Anat. 1999;194(2):303-7. doi: 10.1046/j.1469-7580.1999.19420303.x

5. Guerra RD, Reis PF, de Andrade Bastos A, Brito JC, dos Santos Silva JR, Aragão AJ. Anatomical Study on the Psoas Minor Muscle in Human Fetuses. Int $\mathrm{J}$ Morphol. 2012;30(1):136-9.

6. Khalid S, Iwanaga J, Loukas M, Tubbs RS. Split Femoral Nerve Due to Psoas Tertius Muscle: A Review with Other Cases of Variant Muscles Traversing the Femoral Nerve. Cureus. 2017;9(8):e1555. doi: 10.7759/cureus.1555.

7. Mori M. Statistics on the Musculature of the Japanese. Okajimas Folia Anat Jpn. 1964;40(3):195-300. doi: 10.2535/ofaj1936.40.3_195

8. Seib GA. Incidence of the $m$. psoas minor in man. Am J Phys Anthropol. 1934;19(2):229-46. doi: 10.1002/ajpa.1330190222 